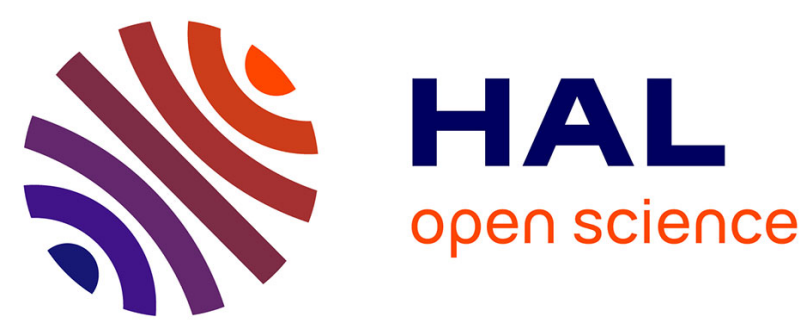

\title{
Transition from core-shell to Janus chemical configuration for bimetallic nanoparticles
}

Cyril Langlois, Z.L. Li, Jinjiang Yuan, Damien Alloyeau, Jaysen Nelayah, Davide Bochicchio, Riccardo Ferrando, Christian Ricolleau

\section{To cite this version:}

Cyril Langlois, Z.L. Li, Jinjiang Yuan, Damien Alloyeau, Jaysen Nelayah, et al.. Transition from coreshell to Janus chemical configuration for bimetallic nanoparticles. Nanoscale, 2012, 4 (11), pp.3381. 10.1039/C2NR11954D . hal-02161350

\section{HAL Id: hal-02161350 \\ https://hal.science/hal-02161350}

Submitted on 20 Jun 2019

HAL is a multi-disciplinary open access archive for the deposit and dissemination of scientific research documents, whether they are published or not. The documents may come from teaching and research institutions in France or abroad, or from public or private research centers.
L'archive ouverte pluridisciplinaire HAL, est destinée au dépôt et à la diffusion de documents scientifiques de niveau recherche, publiés ou non, émanant des établissements d'enseignement et de recherche français ou étrangers, des laboratoires publics ou privés. 


\title{
Transition from core-shell to Janus chemical configuration for bimetallic nanoparticles
}

\author{
Cyril Langlois*a, Ziyou Li ${ }^{\mathrm{b}}$, Jun Yuan ${ }^{\mathrm{c}}$, Damien Alloyeau ${ }^{\mathrm{a}}$, Jaysen Nelayah ${ }^{\mathrm{a}}$, Davide Bochicchio ${ }^{\mathrm{d}}$, \\ Riccardo Ferrando ${ }^{\mathrm{d}}$ and Christian Ricolleau ${ }^{\mathrm{a}}$
}

\author{
${ }_{5}$ Received (in $\left.X X X, X X X\right)$ Xth $X X X X X X X X X 20 X X$, Accepted Xth $X X X X X X X X X 20 X X$ \\ DOI:
}

In order to determine the possibilities to control the chemical configuration of bimetallic nanoparticles, we have considered $\mathrm{CuAg}$ nanoparticles synthesized by physical route as a model in this study. The synthesis was made by pulsed laser deposition under ultra-high vacuum conditions, via a sequential 10 deposition procedure. We show that the temperature of the substrate and the absolute quantity of Ag in a particle are the main parameters that drive the chemical configuration. To explain the transition from a core-shell configuration to a Janus configuration as a function of Ag quantity, we have conducted densityfunctional theory calculations and atomistic molecular dynamics simulations to investigate the stability of this system. The results are presented together with the experimental observations.

\section{1. Introduction}

Bimetallic nanoparticles have been a subject of numerous studies due to the increased capabilities for tuning their properties. Compared to monometallic nanoparticles, they have additional degrees of freedom to adjust the magnetic, optical and catalytic ${ }_{20}$ properties, by playing with the composition and the chemical configuration. ${ }^{1-4}$ Bimetallic nanoparticles can adopt either alloyed or segregated configurations. The alloyed configuration can be either ordered or disordered, accompanied by very different properties. For instance, ordered CoPt nanoparticles present a 25 tetragonal structure which exhibits a high magnetocrystalline anisotropy, whereas disordered nanoparticles are face centered cubic and do not exhibit such anisotropy. ${ }^{5-7}$ Another example, in the field of catalysis, is the use of Ru-Pt nanoparticles to remove carbon monoxide from hydrogen obtained from reformatted 30 hydrocarbons. The article from Alayoglu et al. illustrates perfectly the distinct properties of particles with different chemical configuration. ${ }^{8}$ Core-shell nanoparticles allow to initiate the selective oxidation reaction at lower temperatures compared to their alloyed counterpart, by combining surface strain effect 35 and a modification of the electronic structure of the Pt shell.

Many different segregated chemical arrangements have been identified, including core-shell, onions-like and side-segregated. ${ }^{9}$ The latter is referred as Janus particles because of the similarities between this structure, made of two juxtaposed metallic phases, 40 and the two faces of the Roman god symbolizing 'transitions'. These segregated configurations bring new possibilities for obtaining multifunctional particles. ${ }^{10}$ One unique object can easily present both magnetic and optical properties. For instance Co-Ag core-shell particles have magnetic properties due to the ${ }_{45} \mathrm{Co}$ core, while as the same time exhibit a plasmon resonance associated to the $\mathrm{Ag}$ shell that can be tuned by varying the ratio between the shell thickness and the core diameter. ${ }^{11}$ For the Janus configuration, the idea is no more to protect the core from external reactions. On the contrary, both metals are accessible for 50 interactions with the environment, which makes possible the design of a new class of catalysts. Such adjustments of the properties need a precise control of the structure of the particles and especially of their chemical configuration. This requires significant efforts for controlling the growth of the nanoparticles,

55 either by chemical or physical routes.

It is of fundamental interest to study under which conditions a core-shell growth is favored compared to the Janus configuration. In the present study, we have chosen the $\mathrm{Cu}-\mathrm{Ag}$ system as a model system to determine the possibilities to control the 60 chemical configuration using a physical route. The synthesis is made by pulsed laser deposition under ultra-high vacuum conditions, via a sequential deposition procedure. We show that this particular growth technique is perfectly adapted to obtain segregated bimetallic nanoparticles, with the temperature of the ${ }_{65}$ substrate and the absolute quantity of $\mathrm{Ag}$ in a particle as the main parameters that drive the chemical configuration. To explain the transition from a core-shell configuration to a Janus configuration as a function of $\mathrm{Ag}$ quantity, we have conducted densityfunctional theory (DFT) calculations and atomistic molecular 70 dynamics simulations to investigate the stability of this system. The results will be presented here together with the experimental observations.

\section{Experimental Methods}

$\mathrm{CuAg}$ nanoparticles were prepared by pulsed laser ablation in a 75 high vacuum deposition chamber, under a residual pressure of 10

${ }^{8}$ Torr. A KrF excimer laser at $248 \mathrm{~nm}$ was used for the synthesis, 
with a pulse duration of $25 \mathrm{~ns}$ and a repetition rate of $5 \mathrm{~Hz}$. The energy of the pulses was adjusted separately for $\mathrm{Ag}$ and $\mathrm{Cu}$ depending on their respective ablation threshold. The pulse energy of the laser was $160 \mathrm{~mJ}$ for $\mathrm{Ag}$ and $210 \mathrm{~mJ}$ for $\mathrm{Cu}$. The 5 resulting deposition rates were typically $0.14 \mathrm{~nm}$ per minute for $\mathrm{Cu}$ and $0.5 \mathrm{~nm}$ per minute for Ag. Commercial TEM grids supporting a 10-15 nm thick amorphous carbon film were used as substrate in this study. The temperature of the substrate during the deposition could be adjusted in the range of $20^{\circ} \mathrm{C}$ to $800^{\circ} \mathrm{C}$. ${ }_{10}$ The thickness deposited for a given element was measured by an in situ quartz crystal monitor that gives the nominal thickness in a continuous thin film approximation. For each sample, we carried out $\mathrm{Cu}$ deposition first, immediately followed by $\mathrm{Ag}$ deposition. A $10 \mathrm{~nm}$-thick protective layer of amorphous carbon could be 15 optionally deposited on the sample after cooling down to room temperature. Several Cu@Ag samples were synthesized for this study. The 2@1 samples were obtained by deposition of a nominal thickness of $2 \mathrm{~nm}$ of $\mathrm{Cu}$ followed by deposition of $1 \mathrm{~nm}$ of $\mathrm{Ag}$, with the substrate temperature held at $270^{\circ} \mathrm{C}$ during all the 20 process. With the same scheme, $1 @ 2$ samples were also prepared. The 2@1-100 samples were obtained with the same deposition procedure as for $2 @ 1$ samples, except the temperature of the substrate for $\mathrm{Ag}$ deposition that was decreased to $100^{\circ} \mathrm{C}$. For each synthesis, a reference sample of pure $\mathrm{Cu}$ was grown 25 under the same conditions, referred as $2 @ 0$ sample. When a protective layer of amorphous carbon was deposited, the samples were labeled with the 'ac-' prefix on their name, such as 'ac2@0', 'ac-2@1', and 'ac-2@1-100' respectively.

Several TEM techniques have been employed in this study: 30 conventional Bright Field imaging (BF), Energy Filtered Imaging (EFTEM), High Resolution imaging (HRTEM) and High Angle Annular Dark Field imaging in scanning mode (HAADF-STEM) on two different transmission electron microscopes operating at $200 \mathrm{kV}$ : a JEOL JEM-2100F (University Paris 6, France) and a ${ }_{35}$ FEI Tecnai F20 STEM (University of Birmingham, UK). Elemental maps have been obtained by the three-window technique in EFTEM, and the filtered images at different energies have been aligned using the script developed by Schaffer et al. ${ }^{12,13}$ The EFTEM technique is particularly adapted to study the 40 chemical configurations of bimetallic nanoparticles, as we shown in our previous work. ${ }^{14}$

\section{Results}

3.1. $\mathrm{Cu}-\mathrm{Ag}$ chemical configuration as a function of substrate temperature and deposited thickness

45 HAADF-STEM observations have been carried out on $2 @ 0$ and $2 @ 1$ samples. The microstructural evolution due to the $\mathrm{Ag}$ deposition is clearly evidenced in the two micrographs in Fig. 1, together with the size distributions. Size distribution characteristics and the particle density on the substrate are given 50 in Table 1. For sample 2@0, the pure $\mathrm{Cu}$ nanoparticles show a round shape, characterized by a size distribution on the substrate centered around $10 \mathrm{~nm}$. For sample 2@1, a bimodal distribution is observed with the mean peak positions at 3 and $12 \mathrm{~nm}$. It is worth noting that, apart from the small particles around $3 \mathrm{~nm}$, the 55 particle density on the substrate is significantly lower than for pure $\mathrm{Cu}$ particles, decreasing from $4700 \mu \mathrm{m}^{-2}$ to $3800 \mu \mathrm{m}^{-2}$.
Table 1 Data relative to the particle assemblies of samples 2@0,2@1 and 1 @2 obtained by TEM image thresholding. ${ }^{15}$

60

\begin{tabular}{cccc}
\hline Sample & 2@0 & 2@1 & $1 @ 2$ \\
\hline Data from particle distribution & & & \\
Particles mean diameter $(\mathrm{nm})$ & 9.9 & 12 & 11.8 \\
Peak $F W H M(n m)$ & 5.3 & 8.3 & 6.2 \\
\hline Particle density & & & \\
$\left(\right.$ particles per $\left.\mu \mathrm{m}^{2}\right)$ & 4700 & 3800 & 3700 \\
\hline
\end{tabular}

In order to investigate the chemical configurations that $\mathrm{Cu}$ and $\mathrm{Ag}$ adopt on the substrate after the complete deposition ${ }_{65}$ procedure, EFTEM imaging experiments have been carried out on the $2 @ 1$ sample. Figure 2(a) shows the zero-loss BF image of a representative area on sample $2 @ 1$. The silver chemical map of the same area is presented in Fig. 2(b). A majority of the nanoparticles presents a core-shell configuration, with a mean 70 shell thickness of $1 \mathrm{~nm} \pm 0.5 \mathrm{~nm}$. From this EFTEM chemical map, it is clear that the small particles of $3 \mathrm{~nm}$ are attributed to pure Ag nanoparticles nucleating on their own on the surface, a result confirmed by HRTEM observations. The capping of the $\mathrm{Cu}$ core can be evidenced by Moiré fringes on zone-axis particles 75 and previous work on 2@1 samples using electron tomography showed that the 3D shape of the particles was a truncated sphere with aspect ratio of $1.4{ }^{16}$ Some particles are linked by a silver bridge, showing signs of sintering process at different stages. On Fig. 2(b), the areas labeled $\mathbf{1}$ and $\mathbf{2}$ contain a pair of particles 80 joined together by a silver neck with high curvature, suggesting the initial stage of sintering. Particles labeled 3, 4 and 5 may correspond to a complete sintering, with a convex external shape but isolated copper cores that have not coalesced. The largest core-shell configurations may result from the sintering of ${ }_{85}$ particles with the complete coalescence of the $\mathrm{Cu}$ cores. (a)
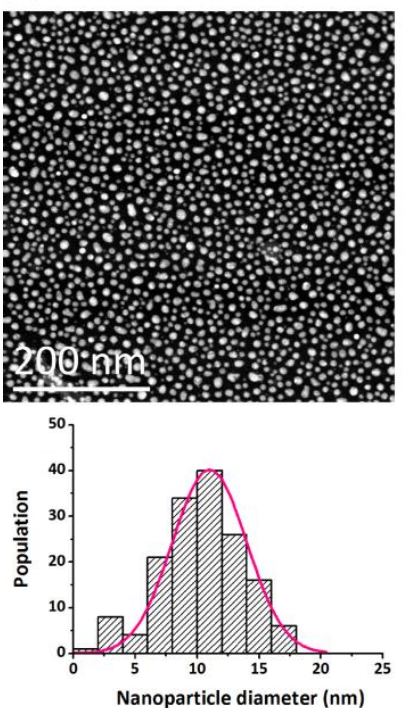

(b)

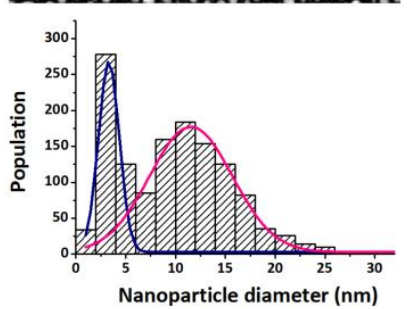

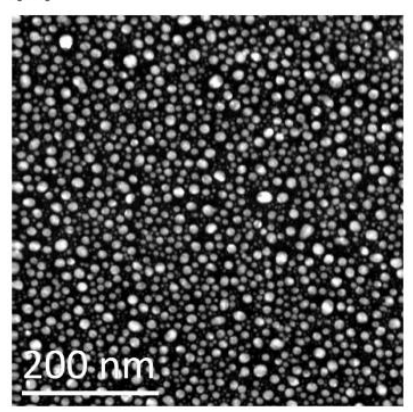

Fig. 1 (Color online) HAADF-STEM images of samples (a) $2 @ 0 \mathrm{Cu}$ and (b) $2 @ 1 \mathrm{CuAg}$. The size distribution for each micrograph is also 90 presented. Characteristics of the distribution are detailed in Table 1. 
The temperature of the substrate can have a strong influence on the chemical configuration of the sample. EFTEM experiments were carried out on 2@1-100 samples. A color chemical map of $\mathrm{Cu}$ and $\mathrm{Ag}$ on a representative area of the sample is presented in ${ }_{5}$ Fig. 3. It is clear that most of the particles are now multi-cores, with $\mathrm{Ag}$ bridging several $\mathrm{Cu}$ cores inside one large ensemble. Though usually two or three $\mathrm{Cu}$ cores are present in one ensemble, up to six $\mathrm{Cu}$ cores per ensemble can also be observed, as pointed out by an arrow in Fig. 3. Small core-shell 10 nanoparticles were also formed on 2@1-100 samples, with a circular projection in the substrate plane that likely corresponds to a direct growth of the shell from $\mathrm{Ag}$ atoms diffusing on the substrate together with those directly landing on the particle. The large multi-core ensembles were also present in sample 2@1, but 15 in very small proportion (see particles labeled 6 on Fig. 2(b)).

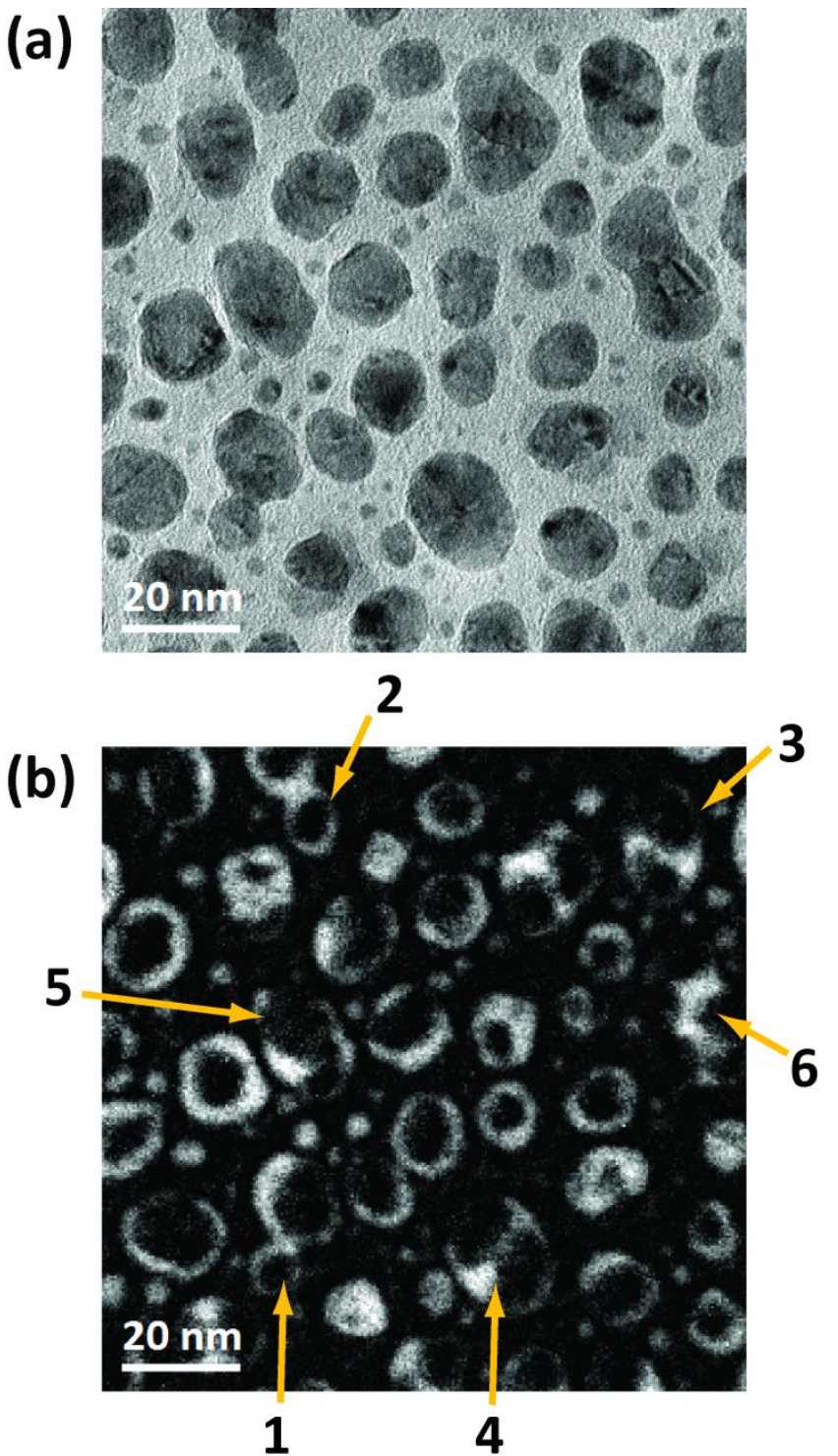

Fig. 2 (Color online) (a) Zero-loss EFTEM image on a representative area 20 of sample 2@1 and (b) Ag elemental map obtained by Energy Filtered TEM on the same area.
To explore the influence of global composition on the chemical configuration of the bimetallic nanoparticles, 1@2 samples have been synthesized. Figure 4 presents the TEM results, with a 25 representative view of the nanoparticles on (a), the size distribution on (b), EFTEM chemical map on (c) and HRTEM on (d). The mean size is around $12 \mathrm{~nm}$ (Table 1), very similar to the 2@1 particles with identical total nominal thickness. On the contrary, it is clear from the chemical map of Fig. 4(c) that the 30 chemical configuration is different from that of sample $2 @ 1$. A majority of bimetallic particles exhibits a $\mathrm{Cu}$ core that is not at the center of the particle, and sometimes touches the outer rim of the particle to form a Janus-like configuration. This is a general tendency over the whole sample, confirmed by high-resolution 35 imaging. A representative example is shown in Fig. 4(d).

\subsection{Thermal stability of CuAg system}

Thermal stability of the $\mathrm{CuAg}$ particles is addressed through annealing experiments under high vacuum of samples ac-2@140100 and 2@1-100, with and without amorphous carbon capping layer respectively. The temperature of the sample was raised to $550^{\circ} \mathrm{C}$ and held for $90 \mathrm{~min}$, to allow for a complete evolution of the particle assembly toward equilibrium. Figure 5 shows the initial and final configurations of the ac-2@1-100 sample. The 45 initial configuration is typical of as-grown sample 2@1-100. The post-synthesis deposition of the amorphous carbon did not alter the particles characteristics. Pure Ag nanoparticles were present between the bimetallic particles, the mean diameter of the latter being around $30 \mathrm{~nm}$. The only change after annealing seems to be 50 the disappearance of the small pure Ag nanoparticles. Most grain boundaries visible in the initial configuration were still there after annealing, indicating little changes within the particles.

The same annealing experiment for the uncapped 2@1-100 sample resulted in more pronounced evolution. Figure 6 shows 55 the particle assembly after annealing, in BF imaging (Fig. 6(a)) and EFTEM imaging (Zero-loss (ZL) image in Fig. 6(b) and Ag elemental map in Fig. 6(c)). The size distribution is given in the inset of Fig. 6(a). The mean size of $32 \mathrm{~nm}$ and the particle density of $720 \mu \mathrm{m}^{-2}$ demonstrate a very strong coalescence between the 60 particles. This phenomenon completely altered the initial structure to larger particles, with a typical Janus chemical configuration, that is, with $\mathrm{Cu}$ on one side of the particle and $\mathrm{Ag}$ on the other side. In most cases, $\mathrm{Cu}$ and $\mathrm{Ag}$ were separated by a unique planar interface crossing the particle. The planar interfaces 65 could also present an angle, as for the particles \#1 in Fig. 6(c).

Here, two distinct structural features are noted: (1) small coreshell nanoparticles were still present on the substrate, under a critical size of $20 \mathrm{~nm}$, and (2) the $\mathrm{Cu}$ part of Janus nanoparticles is still surrounded by a thin $\mathrm{Ag}$ shell up to $3.5 \mathrm{~nm}$ thick. 


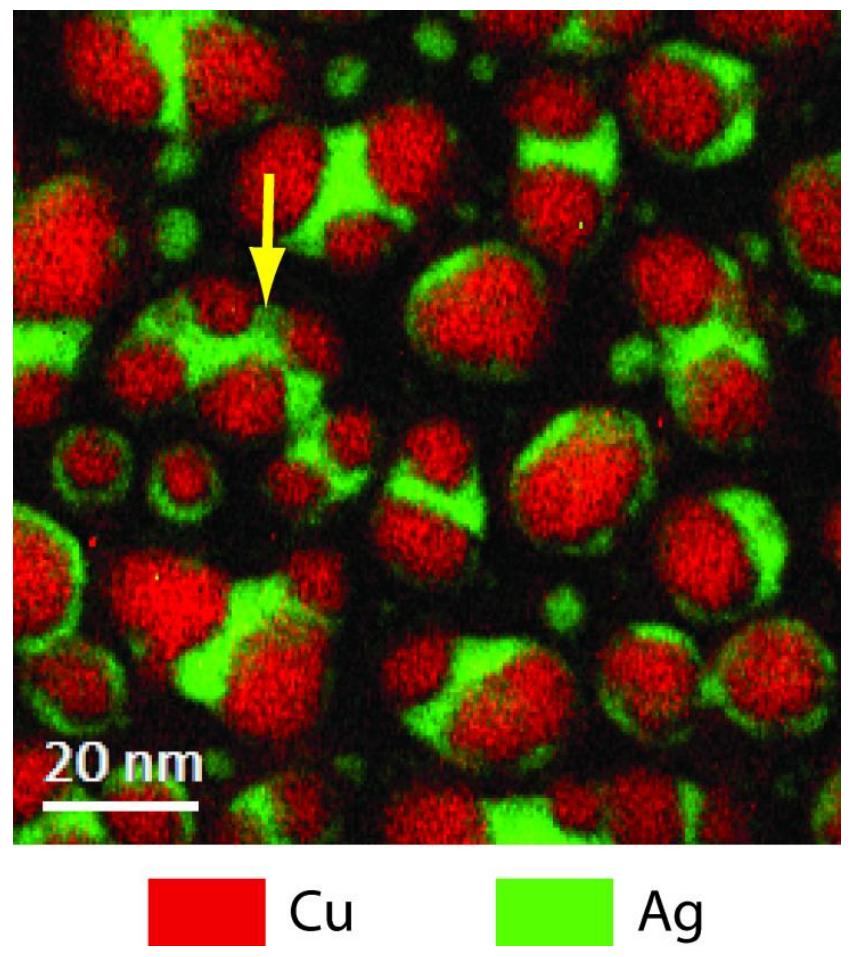

Fig. 3 (Color online) Superimposed $\mathrm{Ag}$ and $\mathrm{Cu}$ elemental map of sample 2@1-100 obtained by EFTEM using the three-window technique. The 5 arrow designs a $\mathrm{CuAg}$ ensemble with six copper cores linked by silver. (a)

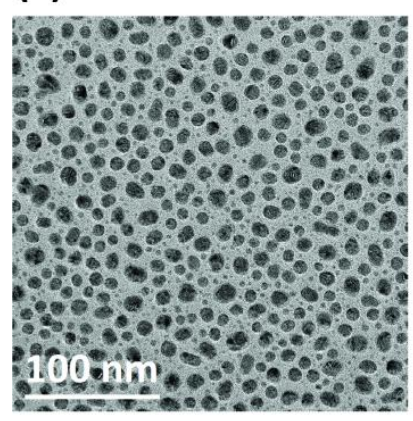

(c)

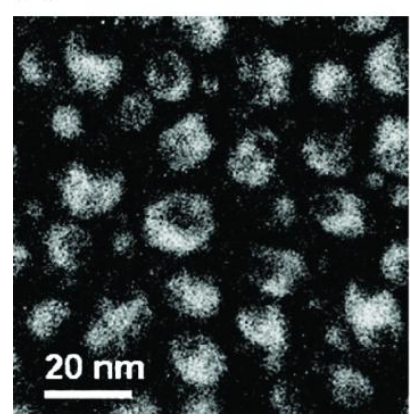

(b)

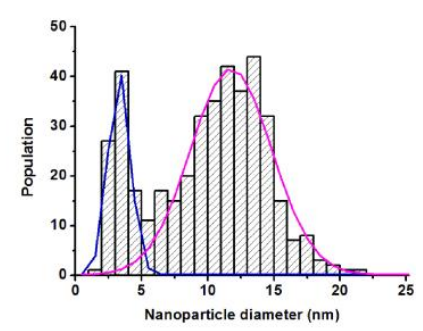

(d)

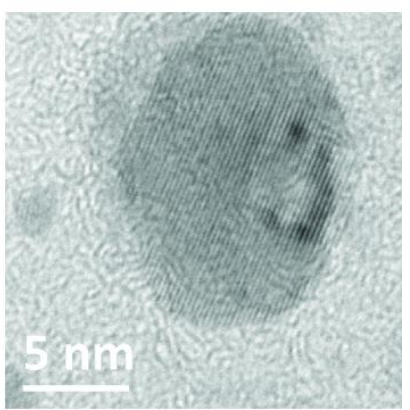

Fig. 4 (Color online) TEM observations of sample 1@2. (a) Bright field TEM image on a representative area of the sample, (b) the size 10 distribution of sample 1@2, (c) Ag elemental map obtained by EFTEM using the three-window technique and (d) HRTEM image of a Janus nanoparticle. (a)

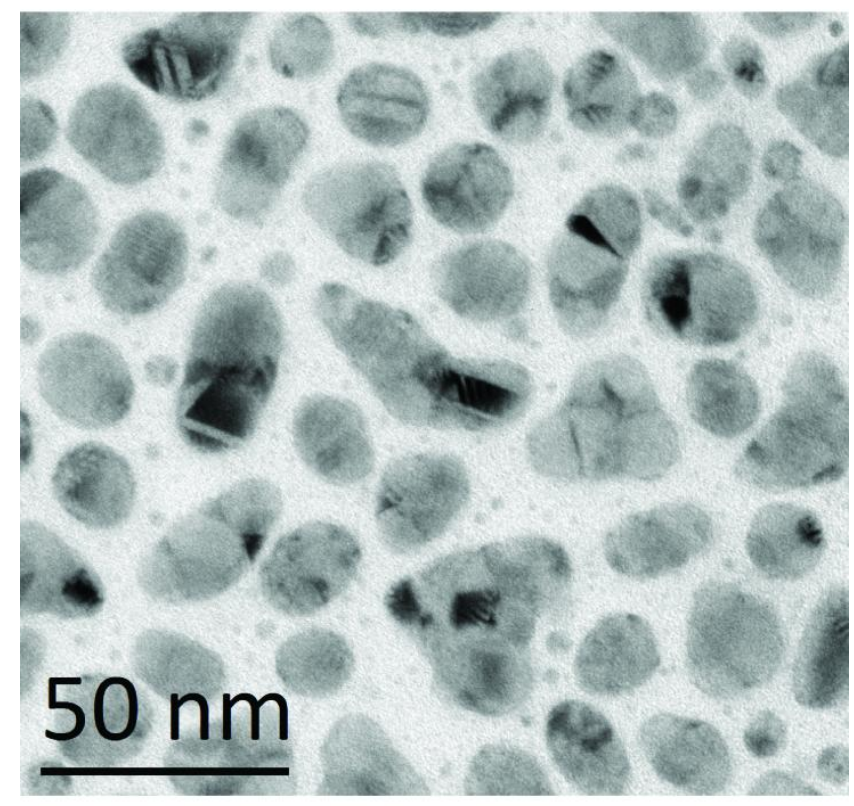

(b)

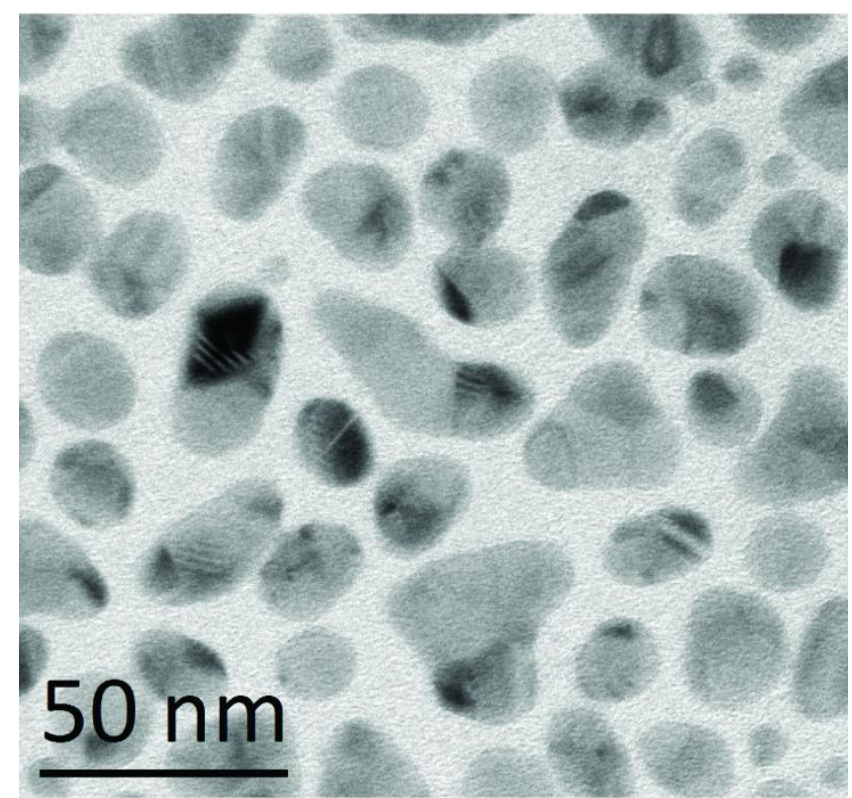

Fig. 5 Annealing experiment on ac-2@1-100 sample: bright field TEM 15 image of (a) the initial configuration and (b) final configuration after 90 $\min$ at $550^{\circ} \mathrm{C}$

\section{Discussion}

\subsection{Growth mechanism of the $2 @ 1$ microstructure}

From the results on samples $2 @ 0$ and 2@1, the mechanisms 20 leading to the structural characteristics can be inferred. The decrease of the particle density from $N_{1}=4700$ to $N_{2}=3800$ particles per squared micron due to silver deposition corresponds roughly to $n=900$ coalescence events per squared micron during the deposition of silver. It means that, assuming only pair25 coalescence events of particles, almost $40 \%$ of the existing $\mathrm{Cu}$ 
particles undergo sintering. Let us consider for simplicity sake that all $\mathrm{Cu}$ particles coalescing are initially identical, with the same size $d_{1}$ (equal to the mean size of the size distribution of 2@0 sample) and an hemi-spherical morphology (see the model 5 on Fig. 7). The coalescence results theoretically in a double-peak size distribution for $\mathrm{Cu}$ cores with peaks positions at $d_{l}$ and $2^{1 / 3} \mathrm{~d}_{1}$. The mean size $d_{2}$ of the CuAg particles for sample $2 @ 1$ should then be:

$$
\begin{aligned}
d_{2} & =\frac{\left(N_{1}-2 n\right) d_{1}+n \sqrt[3]{2} d_{1}}{N_{2}}+2 t_{s} \\
& =d_{1}\left[1+n\left(\frac{\sqrt[3]{2}-1}{N_{1}-n}\right)\right]+2 t_{s}
\end{aligned}
$$

10 with $t_{s}$ the Ag shell thickness measured after coalescence. The second term inside the brackets corresponds to the increase percentage compared to the initial size, that is $6 \%$. This leads to a theoretical increase of the mean size to $d_{2}=12.6 \mathrm{~nm}$, which corresponds nicely to the effective mean size of the particles in 15 the size distribution on sample 2@1 (see Table 1). On the size distribution, we of course do not see the two theoretical peaks because of the polydispersity of the size distributions. From this analysis, the most probable growth mechanism of the $2 @ 1$ coreshell nanoparticles is the formation of an $\mathrm{Ag}$ shell around each 20 copper nanoparticles, with pair-coalescence for $\sim 40 \%$ of the particles to give larger core-shell nanoparticles.

One possible driving force for the coalescence to happen is through diffusion of the metallic species on the substrate. To get insight on the first steps of the initial core-shell structure 25 formation, the diffusion of silver and copper on the carbon substrate were lowered, by reducing the substrate temperature for the $\mathrm{Ag}$ deposition from $270^{\circ} \mathrm{C}$ to $100^{\circ} \mathrm{C}$. Firstly, we observed that the size distribution and particle density of the $\mathrm{Cu}$ cores of the 2@1-100 samples were close to the ones of $2 @ 0$ sample. This 30 means that for the 2@1-100 sample, the temperature of $100^{\circ} \mathrm{C}$ was not sufficiently high to drive the $\mathrm{Cu}$ particle diffusion to lead to their coalescence. Secondly, the Ag elemental map reveals that this temperature was not high enough to cause the atomic rearrangement of the incoming $\mathrm{Ag}$ atomic species around the $\mathrm{Cu}$

35 particles. This effect could be related to the high silver deposition rate of $0.5 \mathrm{~nm}$ per minute. Here, we can conclude that, at $100^{\circ} \mathrm{C}$, the pair-coalescence of particles originates from $\mathrm{Ag}$ bridges formed directly during the deposition of silver.

40 It is worth noting that for other substrates, similar results will be obtained provided that the following general criterion is fulfilled: interactions between the metallic species considered and the substrate must be weak. This is the key point to obtain particles instead of a thin film in the first step of the growth process and to 45 attract the second metallic species around the already formed particles in the second step of the growth. Different metals can exhibit different behavior when interacting with the same substrate. ${ }^{17}$ However, the positive point is that an increase of substrate temperature during the growth can overcome $50 \mathrm{metal} / \mathrm{substrate}$ interactions to a certain extent and leads to the same kind of nanoparticles.

(a)

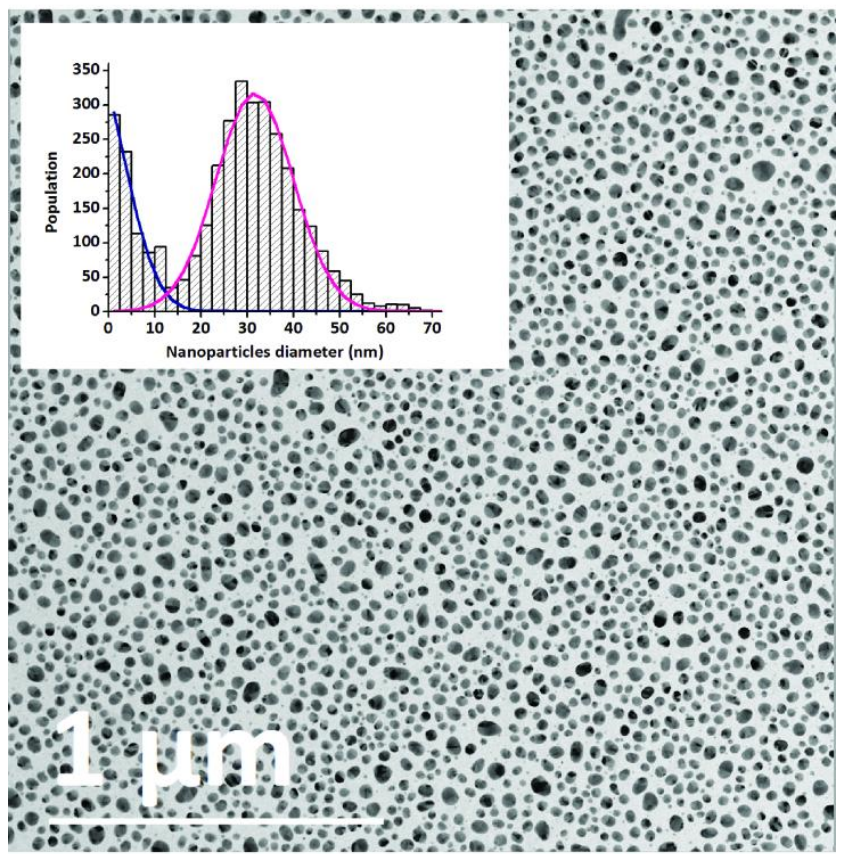

(b)

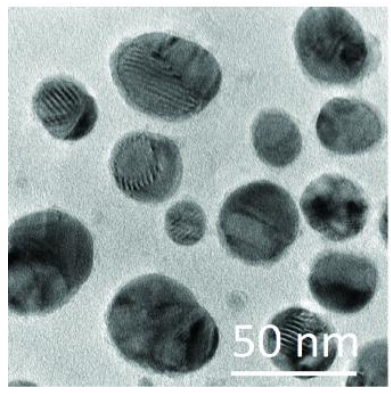

Fig. 6 (Color online) Annealing experiment on 2@1-100 sample. (a) Bright field TEM image of the final configuration of the bimetallic 55 nanoparticles. The inset shows the size distribution of the particles. (b) Zero-loss EFTEM image on a representative area of sample 2@1-100 sample and (c) superimposed Ag and Cu elemental map of sample 2@1100 obtained by EFTEM using the three-window technique on the same area. Color code identical to Fig. 3. ${ }^{12,13}$

60

\subsection{Diffusion mechanisms in the bimetallic $\mathrm{Cu}-\mathrm{Ag}$ nanoparticles}

It is clear from the annealing results that a capping layer of amorphous carbon completely blocks any coalescence of the ${ }_{65}$ particles on the substrate, only allowing the small Ag particles to dissolve themselves in the carbon matrix by Ostwald ripening mechanism. Moreover, no internal re-organization of $\mathrm{Cu}$ and $\mathrm{Ag}$ inside an initial ensemble was noticed. The latter observation is important because theoretically, the temperature was high enough 70 to induce a chemical re-arrangement by volume diffusion mechanisms of $\mathrm{Cu}$ and $\mathrm{Ag}$ in the particles. ${ }^{18}$ If no re-arrangement is observed, it means that the presence of carbon at the surface blocks the mechanisms responsible for the atomic displacements inside a particle. We could deduce from this that it is mainly the 75 diffusion on the surface of the particle that drives any evolution 
of the chemical configuration in the $\mathrm{CuAg}$ particles. Another reason could be also that the surface energy of the particles changes due to the carbon matrix, impeding the driving force toward internal re-arrangement with temperature. ${ }^{19,20}$ On the 5 contrary, the annealing has a strong effect on the uncapped particles that are free to undergo Ostwald ripening and to coalesce through facile diffusion on the substrate. ${ }^{21}$ Without the carbon layer, the coalesced particles can change their chemical arrangement toward Janus configuration. These conclusions are 10 in good agreement with previous simulations carried out on the $\mathrm{Cu}-\mathrm{Ag}$ system in which the authors show that the surface diffusion processes are energetically favored compared to diffusion inside the core. ${ }^{22}$
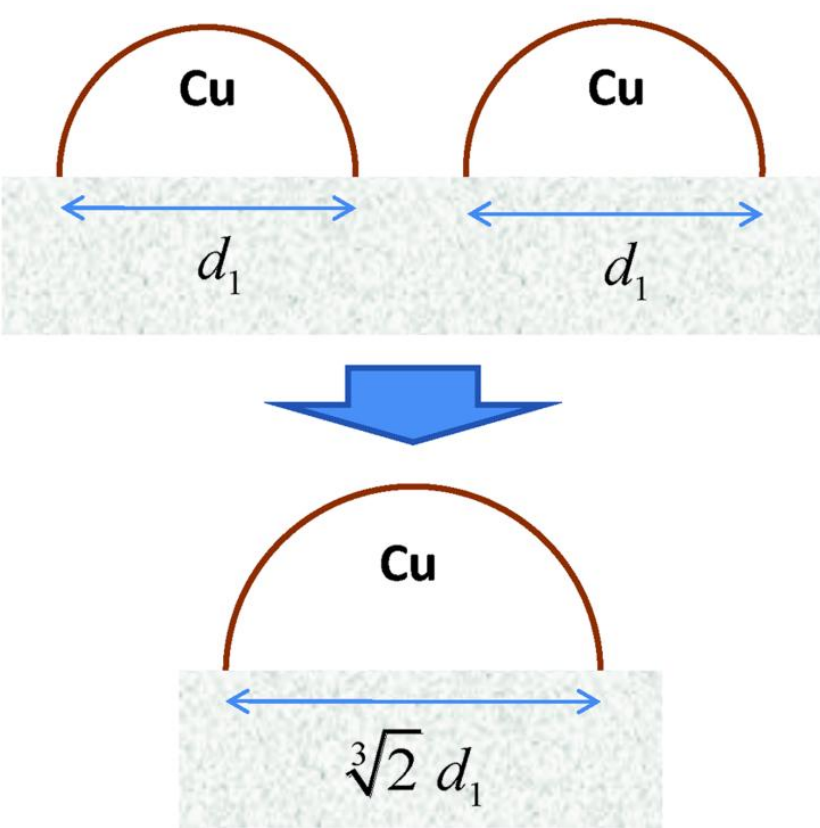

15 Fig. 7 (Color online) Schematic model showing the result of coalescence of two identical hemispheric $\mathrm{Cu}$ particles, with conservation of the total volume of $\mathrm{Cu}$ and the hemispherical morphology.

It seems also important to underline that oxidation hardly affects 20 the nanoparticles. The convergence of several strong arguments allows us to rule out the formation of any oxide in the particle, at least in a significant way:

- all the EFTEM experiment on the oxygen threshold that we carried out show very low signal-to-noise ratio and do not evidence the presence of oxide. EELS spectra do not exhibit the typical fine structure of oxidised $\mathrm{Cu}$ neither.

- all the HRTEM images acquired on these samples are free of any $\mathrm{Cu}$ or $\mathrm{Ag}$ oxide lattice fringes, and often show perfect epitaxy between the two metals.

30 - in case of oxidation of copper, an inversion of the core-shell configuration would be expected, with $\mathrm{Ag}$ in the core and $\mathrm{Cu}$ oxide as a shell because the mean surface energy of $\mathrm{Cu}_{2} \mathrm{O}$ is less than the one of $\mathrm{Ag} .{ }^{23}$

- we never noticed any difference between carbon-capped and

$35 \quad$ air-exposed 2@1 sample.

\subsection{Competition between core-shell and Janus chemical configuration}

40 It is interesting to note that, although the $2 @ 1$ and $1 @ 2$ samples are very similar from the point of view of synthesis apart from their chemical composition, the EFTEM characterization reveals a different internal structure of $\mathrm{CuAg}$ particles. For the 1 @2 sample, not only the average $\mathrm{Ag}$ shell thickness is larger as the ${ }_{45} \mathrm{Ag}$ content increases, but also the core is off-centred and very close to the rim of the particle. These results support the idea that the increase in shell thickness is responsible for the evolution of the chemical configuration toward Janus configuration. This hypothesis can be verified by the annealing experiments. In our 50 annealing experiments, the amount of matter was kept constant (equivalent to $2 @ 1$ samples) but coalescence events occurred, resulting in larger particles, hence larger core radius and a larger amount of silver available for re-arrangement around the $\mathrm{Cu}$ core. This suggests that both an increase of $\mathrm{Ag}$ concentration and a 55 coalescence event have a consequence in common, that is an increase of the amount of silver for a given $\mathrm{CuAg}$ particle. Therefore, the Janus configuration identified for the annealed samples may originate from a larger shell thickness that turns finally to the side-segregated configuration, as for the $1 @ 2$ ${ }_{60}$ samples. From the measurement on the annealed sample and the $1 @ 2$ sample, the critical thickness $t_{c}$ at which the transition occurs from the core-shell to the Janus configuration is estimated to be 3-4 nm. Because of the large size distribution of the particles in the samples, we always find both type of chemical ${ }_{65}$ configurations in a given sample. This result is confirmed by measurements carried out on larger $\mathrm{CuAg}$ nanoparticles, synthesized by thermal evaporation in a previous work. ${ }^{14}$ These particles correspond to a $6 @ 3$ system. Both Janus and perfect core-shell nanoparticles are present on the substrate, but for the 70 perfect core-shell configuration, there are no shell with a thickness greater than $4 \mathrm{~nm}$, independently of the $\mathrm{Cu}$ core size.

To gain further insight of the driving force for the transition from the core-shell to Janus configuration, we carried out DFT calculations and molecular dynamics simulations (MD) using 75 atomistic potentials derived from second-moment approximation to the tight-binding model. DFT calculations have been made by using the Perdew-Burke-Ernzerhof exchange-correlation functional (PBE) and the Quantum Espresso code. ${ }^{24}$ Details are given in the work from Bochiccio et al.. ${ }^{25}$ We report atomistic ${ }_{80}$ calculations by means of two different parameter sets of the Gupta potential. ${ }^{26}$ Parametrization P1 has been fitted on DFT calculations of small $\mathrm{Ag}-\mathrm{Cu}$ clusters, ${ }^{25}$ whereas parametrization P2 was fitted on the experimental energetics of single impurities of the bulk host matrix of the other metal. ${ }^{22}$ These atomistic ${ }^{85}$ potentials have been thoroughly tested against DFT calculations, which showed that P1 agrees very well for what concerns the energetics of core-shell nanoparticles. The agreement of P2 is slightly worse, but the qualitative behaviour is the same for both P1 and P2 (see the supplementary online material of Ref. 90 Bochicchio et al. $^{25}$ ). We have calculated the energetics of single $\mathrm{Cu}$ impurities placed inside truncated octahedral Ag clusters (see Fig. 8) in order to determine the most favorable position inside the cluster. We have considered three cluster sizes: 79, 201 and 586 atoms. For the smallest cluster, we performed both DFT and 95 atomistic potential calculations. For 201 and 586 atoms, DFT 
calculations become very cumbersome, so that only atomistic calculations were performed. The results are presented in Table 2, with the energies associated to $\mathrm{Cu}$ positions under different types of facets. For the $\mathrm{Ag}_{78} \mathrm{Cu}_{1}$ the DFT calculations are in good 5 agreement with the results of $\mathrm{P} 2$ potential, both showing that for the $\mathrm{Cu}$ impurity, the sub-surface positions, just one layer below the surface, are always more stable than central and surface positions. Potential P1 does not agree with DFT and P2 for this size, because it prefers the central positions. For $\mathrm{Ag}_{200} \mathrm{Cu}_{1}$ and ${ }_{10} \mathrm{Ag}_{585} \mathrm{Cu}_{1}, \mathrm{P} 2$ confirms the tendency to favor sites that are just one layer below the surface. Also P1 does not favor the central sites anymore when size increases. According to $\mathrm{P} 1$, the most favorable sites for $\mathrm{Cu}$ impurities are located two layers below the surface. Our previous work on the simulation of the $\mathrm{Cu}-\mathrm{Ag}$ 15 system has shown that, when $\mathrm{Cu}$ atoms are deposited on a $\mathrm{Ag}$ cluster (inversed deposition model), the $\mathrm{Cu}$ atoms agglomerates under the surface in order to form a pure $\mathrm{Cu}$ cluster. ${ }^{27}$
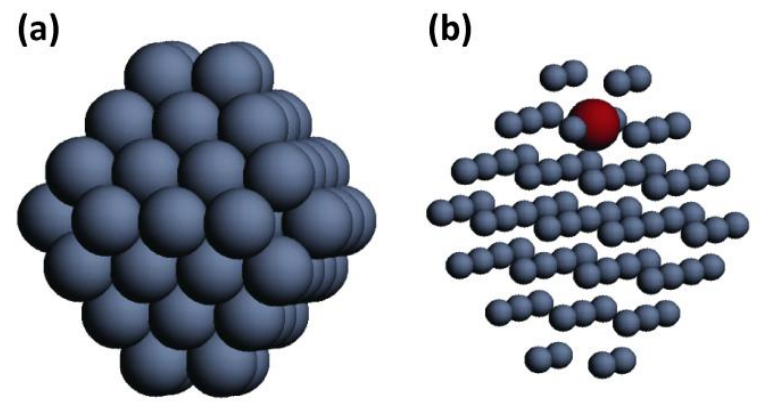

Fig. 8 (Color online) Model for the truncated octahedral $\mathrm{Ag}_{78} \mathrm{Cu}_{1}$ cluster 20 used in the DFT, P1 and P2 calculations. The external surface of the $\mathrm{Ag}_{78} \mathrm{Cu}_{1}$ cluster is represented in (a). The cluster configuration in (b) represent the $\mathrm{Cu}$ atom in the most favorable place according to DFT calculations.

The energy differences between these positions in the cluster are 25 small, so that zero-point energy contribution may be important. ${ }^{28}$ However, when the concentration of impurities is finite, the energy differences between aggregates in central and subsurface positions greatly increases. For example, we have considered the truncated octahedron of 201 atoms with $13 \mathrm{Cu}$ atoms. According 30 to $\mathrm{P} 2$, the best subsurface position of the $\mathrm{Cu}$ aggregate is lower by about $1 \mathrm{eV}$ than the central position of the aggregate. On the contrary, an $\mathrm{Ag}$ impurity in a $\mathrm{Cu}$ cluster would prefer lowcoordination sites, such as vertex sites on the surface. We have verified this for the truncated octahedron of 201 atoms, in which 35 the vertex site is favoured by $0.9 \mathrm{eV}$ compared to central and subsurface sites.

All these results are consistent with the formation of off-center $\mathrm{Cu}$ aggregates in Ag-rich clusters. If we consider only the composition inside the particle, it is clear that a low concentration 40 of $\mathrm{Ag}$ (i.e. thin $\mathrm{Ag}$ shell) corresponds to a core-shell configuration (as clearly shown by the global optimization results $^{25}$ ) whereas a large concentration of $\mathrm{Ag}$ (i.e. a thick $\mathrm{Ag}$ shell) should correspond to a Janus configuration.

In order to verify this hypothesis, we have performed global45 optimization searches of chemical ordering in Ag-rich clusters. We have considered an fcc truncated octahedron of 586 atoms containing $90 \% \mathrm{Ag}$ and $10 \% \mathrm{Cu}$. We have initially placed the $\mathrm{Cu}$ atoms randomly in the cluster and then we have performed extensive global optimization runs in which only exchange moves 50 between atoms of different species were used. The lowest energy configurations according to $\mathrm{P} 1$ and $\mathrm{P} 2$ are shown in Fig. 9. For both models, an off-center $\mathrm{Cu}$ core is obtained so that a quasiJanus particle is formed. The chemical ordering of these nanoparticles clearly resembles that of the experimental cluster of ${ }_{55}$ Fig. 4(d).

Table 2 Results from DFT and atomistic calculations on $\mathrm{Ag}$ clusters containing a single $\mathrm{Cu}$ atom. For each size, the data report the energy difference from the configuration with $\mathrm{Cu}$ on ${ }_{60}$ the center of the cluster, which is taken as the zero of energy. Energy differences are given in $\mathrm{eV}$.

\section{Cluster Position of the impurity DFT P1 P2}

\begin{tabular}{clccc}
\hline TO $_{79} \quad$ central sites & 0.000 & 0.000 & 0.000 \\
\hline & & & & \\
\hline 1 layer below (111) facet & -0.001 & 0.035 & -0.046 \\
& & & \\
\hline 1 layer below (100) facet & -0.100 & 0.109 & -0.100 \\
& & & \\
\hline surface sites on (111) facet & 0.136 & 0.312 & 0.100
\end{tabular}

\begin{tabular}{llll}
\hline $\mathbf{T O}_{\mathbf{2 0 1}} \quad$ central sites & 0.000 & 0.000
\end{tabular}

\begin{tabular}{llll}
\hline 1 layer below (111) facet & 0.000 & -0.035 \\
& 1 layer below (100) facet & 0.060 & -0.067 \\
\hline 2 layers below (100) facet & -0.028 & -0.037 \\
\hline $\mathbf{T O}_{\mathbf{5 8 6}}$ central sites & & \\
\hline & & 0.000 & 0.000 \\
\hline 1 layer below (111) facet & & \\
\hline 1 layer below (100) facet & 0.010 & -0.050 \\
& & 0.047 & -0.065 \\
\hline 2 layers below (100) facet & -0.023 & -0.030
\end{tabular}

These simulation results are in good agreement with the ${ }_{65}$ experimental observations on the $\mathrm{Cu}-\mathrm{Ag}$ samples. Moreover, these simulations justify the presence of a thin $\mathrm{Ag}$ layer around the $\mathrm{Cu}$ part for the Janus nanoparticles. Our results agree with a tendency already observed experimentally in surface science for the $\mathrm{Cu}$ growth on $\mathrm{Ag}(111) .{ }^{29}$ In the work from Boquet et al., the 70 authors show that $\mathrm{Cu}$ clusters grown on $\mathrm{Ag}$ (111) surfaces are covered by one monolayer of silver through segregation effects. It 
is worth noting that this result has not been experimentally observed for nanoparticles, even though it is likely to be representative of a quite general behavior. In fact, atomistic simulations are predicting that the same kind of behavior is likely 5 to be observed in $\mathrm{AgCo}$ and $\mathrm{AgNi}$ too. ${ }^{30,31}$

In order to have a complete view of the problem, it is important to consider also the surface science literature on the $\mathrm{CuAg}$ system. A brief review shows that the growth modes of $\mathrm{Ag}$ on $\mathrm{Cu}$ or $\mathrm{Cu}$ on $\mathrm{Ag}$ are very different, they strongly depend on the orientation 10 of the substrate, on the substrate temperature and on the amount of matter deposited. The growth of $\mathrm{Ag}$ on $\mathrm{Cu}$ (111) has been widely studied: although some discrepancy exists, a layer-bylayer growth mode is favoured. ${ }^{32-34}$ Twinning has been observed, resulting in similar heteroepitaxy relationships as for the bulk. ${ }^{35,36}$

15 When the $\mathrm{Cu}$ substrate is [110] oriented, the growth mode is reported to be layer-by-layer at low temperature, and StranskiKrastanov at high temperature. ${ }^{37}$ For the growth of $\mathrm{Cu}$ on $\mathrm{Ag}$, the Stranski-Krastanov growth mode is the most frequently reported. ${ }^{29,38}$ From these experimental data, it is clear that the 20 situation is very complex in the case of a nanoparticle because different facets are open to the growth of the shell. Moreover, the sintering between the particles induced by the deposition of silver in the two-step growth procedure makes the comprehension of the final chemical configuration quite complicated. Another 25 complication arises from the fact that the synthesis by pulsedlaser ablation often results in non-equilibrium configurations driven by kinetic effects. The present work highlights the importance of monodispersed nanoparticles and good control of chemical composition in achieving desired structure in bimetallic 30 nanosystems.
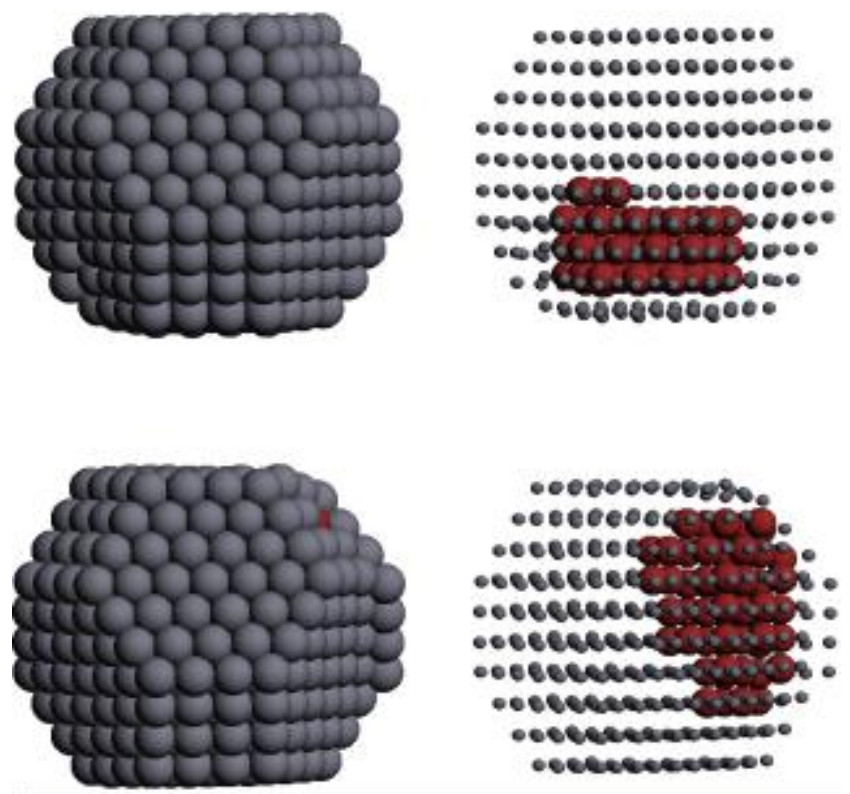

35 Fig. 9 (Color Online) Structure of a truncated octahedron of 586 containing $90 \% \mathrm{Ag}$ and $10 \% \mathrm{Cu}$ after global optimization runs of exchange moves only. The structure is somewhat distorted to release the strain. In the top and bottom row we show the results of P1 and P2, respectively. In both models, quasi-janus configurations are obtained.

\section{${ }_{40}$ 5. Conclusion}

We have presented in this study the synthesis of bimetallic $\mathrm{Cu}-\mathrm{Ag}$ nanoparticles via pulsed laser ablation, with various chemical compositions. We demonstrated that the deposition of silver on pre-existing copper cores (two-step growth procedure) induced a 45 sintering between the particles, if the substrate temperature was high enough to enable surface diffusion on the substrate. The thermal stability of these nanoparticles has also been studied by annealing experiments under high vacuum conditions. $\mathrm{Cu}$ and $\mathrm{Ag}$ adopted a segregated chemical configuration, according to the 50 immiscibility of this alloy and the strong difference of their mean surface energy. Two types of segregated configurations were identified: a core-shell configuration for shell thickness under 3 to $4 \mathrm{~nm}$, and a Janus configuration when the amount of Ag inside a particle was in excess compared to this critical thickness. For 55 the Janus configuration, we determined that the $\mathrm{Cu}$ part of a particle remains covered by a thin $\mathrm{Ag}$ layer. The above observations are supported by DFT calculations and MD simulations, which suggest that the energetically favorite position for a $\mathrm{Cu}$ impurity in an $\mathrm{Ag}$ cluster is a sub-surface position. ${ }_{60}$ These sub-surface impurities can act as seeds for the nucleation of off-center $\mathrm{Cu}$ clusters, and lead to the formation of quasi-Janus structures for Ag-rich compositions.

The present work is a step forward to the understanding of the mechanisms leading to the formation of a particular chemical 65 configuration for bimetallic particles. Further studies on the influence of the chemical configuration on the optical properties of such systems are currently under investigation.

\section{Acknoledgments}

We are grateful to the Egide (France) and British Council 70 (UK) for supporting this study through the Alliance project, as well as the European COST Action MP0903. R.F. and D.B are grateful for support from CINECA supercomputing center for the project HP10CF1XI1. ZY.L. and J.Y. thank supports from UK EPSRC. We are also grateful to Region Ile-de-France for the 75 support to the JEOL $2100 \mathrm{~F}$ electron microscope installed at University Paris 6.

\section{Notes and references}

${ }^{*}$ a Laboratoire Matériaux et Phénomènes Quantiques, CNRS-UMR 7162, 80 Université Paris Diderot - Paris 7, Case Courrier 7021, 75205 Paris

Cedex 13, France.E-Mail : cyril.langlois@insa-lyon.fr

${ }^{b}$ Nanoscale Physics Research Laboratory, School of Physics and Astronomy, University of Birmingham, Birmingham B15 2TT, UK ${ }^{c}$ Department of Physics, University of York, Heslington, York YO10 5DD, 85 United Kingdom

${ }^{d}$ Dipartimento di Fisica, Università di Genova and IMEM/CNR, Via Dodecaneso 33, 16146 Genova, Italy

901 L. Guczi, Catalysis Today, 2005, 101, 53-64.

2 K. Major, C. De and S. Obare, Plasmonics, 2009, 4, 61-78.

3 N. S. Sobal, M. Hilgendorff, H. Mahwald, M. Giersig, M. Spasova, T. Radetic and M. Farle, Nano Letters, 2002, 2, 621-624.

4 H. Zeng, S. Sun, J. Li, Z. L. Wang and J. P. Liu, Applied Physics ${ }_{95}$ Letters, 2004, 85, 792-794. 
5 D. Alloyeau, C. Ricolleau, C. Mottet, T. Oikawa, C. Langlois, Y. Le Bouar, N. Braidy and A. Loiseau, Nature Materials, 2009, 8, 940-946.

6 F. Tournus, N. Blanc, A. Tamion, M. Hillenkamp and V. Dupuis, Journal of Magnetism and Magnetic Materials, 2011, 323, 1868-1872.

57 B. Bian, D. E. Laughlin, K. Sato and Y. Hirotsu, Journal of Applied Physics, 2000, 87, 6962-6964.

8 S. Alayoglu, A. U. Nilekar, M. Mavrikakis and B. Eichhorn, Nature, 2008, 7, 333.

9 R. Ferrando, J. Jellinek and R. L. Johnston, Chemical Reviews, 2008, 10 108, 845-910.

10 Y. Jin, C. Jia, S.-W. Huang, M. O'Donnell and X. Gao, Nat Commun, 2010, 1, 41.

11 M. Liu and P. Guyot-Sionnest, Journal of Physical Chemistry B, 2004, 108, 5882-5888.

1512 L. Reimer, Energy-Filtering Transmission Electron Microscopy, Springer Verlag, Berlin, 1995.

13 B. Schaffer, W. Grogger and G. Kothleitner, Ultramicroscopy, 2004, 102, 27-36.

14 C. Langlois, T. Oikawa, P. Bayle-Guillemaud and C. Ricolleau, 20 Journal of Nanoparticle Research, 2008, 10, 997-1007.

15 Digital Micrograph ${ }^{\mathrm{TM}}$, www.gatan.com/scripting/downloads.php, software by Gatan Inc.

16 M. Valamanesh, C. Langlois, D. Alloyeau, E. Lacaze and C. Ricolleau, Ultramicroscopy, 2011, 111, 149-154.

2517 A. A. Schmidt, H. Eggers, K. Herwig and R. Anton, Surface Science, 1996, 349, 301-316.

18 D. B. Butrymowicz, J. R. Manning and M. E. Read, Journal of Physical and Chemical Reference Data, 1974, 3, 527-602.

19 O. M. Løvvik and S. M. Opalka, Surface Science, 2008, 602, 2840302844.

20 F. Tao, M. E. Grass, Y. Zhang, D. R. Butcher, J. R. Renzas, Z. Liu, J. Y. Chung, B. S. Mun, M. Salmeron and G. A. Somorjai, Science, 2008, 322, 932-934.

21 D. Alloyeau, G. Prévot, Y. Le Bouar, T. Oikawa, C. Langlois, A. 35 Loiseau and C. Ricolleau, Physical Review Letters, 2010, 105, 255901.

22 F. Baletto, C. Mottet and R. Ferrando, Physical Review B, 2002, 66, 155420 .

23 D. A. Firmansyah, T. Kim, S. Kim, K. Sullivan, M. R. Zachariah and D. Lee, Langmuir, 2009, 25, 7063-7071.

4024 P. Giannozzi, S. Baroni, N. Bonini, M. Calandra, R. Car, C. Cavazzoni, D. Ceresoli, G. L. Chiarotti, M. Cococcioni, I. Dabo, A. Dal Corso, S. De Gironcoli, S. Fabris, G. Fratesi, R. Gebauer, U. Gerstmann, C. Gougoussis, A. Kokalj, M. Lazzeri, L. Martin-Samos, N. Marzari, F. Mauri, R. Mazzarello, S. Paolini, A. Pasquarello, L. Paulatto, C. Sbraccia, ${ }_{45}$ S. Scandolo, G. Sclauzero, A. P. Seitsonen, A. Smogunov, P. Umari and R. M. Wentzcovitch, Journal of Physics: Condensed Matter, 2009, 21, 395502.

25 D. Bochicchio and R. Ferrando, Nano Letters, 2010, 10, 4211-4216.

26 R. P. Gupta, Physical Review B, 1981, 23, 6265-6270.

5027 F. Baletto, C. Mottet and R. Ferrando, Physical Review Letters, 2003, 90, 135504.

28 H. Yildirim, A. Kara and T. S. Rahman, Journal of Physics: Condensed Matter, 2009, 21, 084220.

29 F. Bocquet, C. Maurel, J.-M. Roussel, M. Abel, M. Koudia and L. 55 Porte, Physical Review B, 2005, 71, 075405.
30 I. Parsina and F. Baletto, The Journal of Physical Chemistry C, 2010, 114, 1504-1511.

31 F. Calvo, E. Cottancin and M. Broyer, Physical Review B, 2008, 77, 121406.

6032 Y. Borensztein, T. Lopez-Rios and G. Vuye, Physical Review B, 1988, 37, 6235 .

33 U. Kürpick, G. Meister and A. Goldmann, Applied Surface Science, 1995, 89, 383-392.

34 C. Mottet, G. Tréglia and B. Legrand, Physical Review B, 1992, 46, 6516018.

35 J. B. Liu, Y. W. Zeng and L. Meng, Journal of Alloys and Compounds, 2008, 464, 168-173.

36 K. Umezawa, S. Nakanishi, M. Yoshimura, K. Ojima, K. Ueda and W. M. Gibson, Physical Review B, 2000, 63, 035402.

7037 T. N. Taylor, R. E. Muenchausen and M. A. Hoffbauer, Surface Science, 1991, 243, 65-82.

38 P. A. Huttunen and A. Vehanen, Physical Review B, 1990, 42, 11570. 